\title{
Crueldad, paternalismo y estilo tragicómico en Memorias póstumas de Blas Cubas de Joaquín María Machado de Assis
}

Francisco Ramírez Santacruz

Mâo e a Luva (1874), Helena (1876) e Iaiá Garcia (1878), tres de
las cuatro primeras novelas de Joaquín María Machado de Assis, el novelista brasileño más importante del siglo XIX, se ocupan del problema del paternalismo en el terreno de las relaciones amorosas y de la humillación de las mujeres que esta situación conlleva. ${ }^{1}$ Por lo mismo no sorprende que en dos ocasiones dichas obras hagan una referencia explícita a la mujer en el título. En efecto, Machado de Assis, sin par en el ámbito latinoamericano de su tiempo, se ocupó constantemente del problema de la relación entre la clase dominante y la clase libre (pero sin trabajo) partiendo de la figura femenina, poniendo así sobre la mesa uno de los problemas claves, pero prohibidos, de su época. En su quinta novela, Memorias póstumas de Blas Cubas (1880), nuestro autor inaugura el realismo en Brasil y dedica espléndidas páginas al tema de marras. Blas, el protagonista, quien

\footnotetext{
${ }^{1}$ La primera novela de Machado de Assis, Ressurreição (1872), trata sobre una relación amorosa que no culmina frente al altar debido a razones infundadas del novio. Sobre las protagonistas de estas tres novelas apunta Schwarz: "As heroínas são moças nascidas abaixo do seu merecimento, e tocará às famílias abastadas eleválas, reparando o 'equívoco"” (1992: 67).
} 
narra desde ultratumba, pertenece a la clase dominante y se relaciona con tres mujeres de una clase social inferior a la suya (Eugenia, Marcela y Loló) y con una de su misma clase social (Virgilia). El análisis de las relaciones entre Blas y sus mujeres evidencia cómo Machado de Assis se sirvió de un problema social brasileño del siglo XIX para dotar de unidad interna una obra, cuya estructura parece rehuir cualquier intento de homogeneidad, además de hacer una tragicómica crítica social de su época.

La historia de Eugenia (caps. 30-36 y 158) conforma uno de los episodios más representativos del paternalismo no sólo por el sitio que ocupa dentro de la estructura, sino, sobre todo, porque establece el paradigma de las siguientes relaciones de Blas con mujeres de una clase social inferior a la suya. Después de aceptar la propuesta de su padre para bajar a Río y abandonar la Tijuca, donde se refugió tras la muerte de su madre, el narrador decide visitar a doña Eusebia, una antigua conocida a la que de chico le jugó una broma bastante pesada. En su casa, doña Eusebia le presenta a Blas a su hija Eugenia. De inmediato, Blas escoge para Eugenia, de dieciséis años de edad, un mote despectivo, "la flor de la mata" (81), alusivo a su posible concepción dentro de una relación clandestina de su madre. Obviamente, el narrador espera que su lector sea consciente del significado etimológico del nombre "Eugenia" (la "bien nacida" o "noble"), pues de esto Eugenia no tiene mucho a los ojos siempre mordaces de Blas. Durante su visita, Blas se percata de que Eugenia padece de un defecto físico, lo que le provoca un dilema moral: “¿Por qué bonita, si coja? ¿Por qué coja, si bonita?” (82). Pese a estas primeras reflexiones, Blas inicia con la chica un efímero romance, regido por el signo de la crueldad. El protagonista humilla sistemáticamente a Eugenia, utilizando su defecto físico como pretexto. Después de varios días de sadismo, Blas se aleja de su refugio y baja a Río. Ya casi al final de su vida (cap. 158), Blas se cruza, de nueva cuenta, con Eugenia, que vive de pedir limosna y en la miseria. Nunca más vuelve a saber de ella. 
En "The Historical Meaning of Cruelty in Machado de Assis", Roberto Schwarz lleva a cabo un sesudo análisis del episodio de Eugenia y advierte que la particularidad sociológica del "romance" no debe ser disuelto en el arquetipo de una relación entre hombre rico $\mathrm{y}$ una joven pobre, pues se hace necesario estudiar las estructuras sociales de la época y sus conflictos para comprender la relación entre Blas y Eugenia:

As a result of slavery, there was no developed job market in Brazil; in other words, no practical structure existed in which an individual without means might gain autonomy. A person's value therefore depended on arbitrary (and humiliating, if inconsistent) recognition by a wealthy patron. In this context, it does not seem unreasonable to suggest that Eugênia, among other similar characters, embodies the general reality of the poor, free person in Brazilian slave society (1996: 167-168).

Esta realidad de la persona libre (pero pobre) de la que habla Schwarz en la sociedad esclavista brasileńa significa la experiencia de una libertad relativa, que depende de los favores que algún protector rico quiera otorgar. Visto así, la relación entre Eugenia y Blas aparece bajo una luz doblemente cruel. En dos ocasiones, Eugenia, en lugar de aceptar la diferencia social de clase entre ella y Blas, si no la rechaza, por lo menos, la cuestiona considerablemente, provocando que el narrador se sienta afrentado. Eugenia pone en crisis la visión del mundo de Blas al colocar el énfasis de la relación en el aspecto de la dignidad interior y no en la exterioridad materialista. ${ }^{2}$ Por consiguiente, el definitivo rechazo de Blas hacia Eugenia no nace a raíz de su defecto físico, sino que es determinado por la lógica de las diferencias de clase:

${ }^{2}$ Analizo el caso con mayor detalle cuando estudio la relación entre Loló y Blas, véase nota 10 . 
These positive qualities, which attract the young man, become negative attributes when, accompanied by poverty, they create a moral and sentimental dilemma for him. Let us keep three points in mind: (a) the crux of the matter is class, and the physical defect is a mere afterthought that functions as an alibi; (b) in the context of class domination, the inferior class's human achievements are seen as further misfortunes (Schwarz, 1996: 172-173).

De allí que Blas sentencie fría y calculadoramente: “Tú, mi Eugenia, no te descalzaste nunca; fuiste por la calle de la vida cojeando de la pierna y del amor, triste como los entierros pobres, solitaria, callada, laboriosa, hasta que viniste también a esta otra orilla. Lo que no sé es si tu existencia fuese muy necesaria al siglo" (86).

A partir de estas consideraciones podemos acercarnos a las posteriores relaciones de Blas con Marcela y Loló, donde las diferencias sociales también determinan el desarrollo y fin de los romances. Asimismo precisa observar cómo Machado de Assis introduce ecos del episodio de Eugenia en las otras relaciones, lo que posibilita una correspondencia de temas y motivos a lo largo de toda la novela.

Marcela es el primer amor de Blas, quien inicia su relación con ella a partir de un capricho de juventud. A la edad de diecisiete años, Blas se cruza en la calle con una mujer de extraordinaria belleza de quien queda prendido. Poco después su tío Juan le propone ir a una fiesta nocturna que se celebra, por azares del destino, en casa de la bella mujer que Blas admiró unos días antes, cuyo nombre es Marcela. Durante todo el festejo, Blas no le quita de encima los ojos a la anfitriona y desinhibido por los efectos del vino besa a Marcela sin que ésta oponga resistencia. Marcela y Blas comienzan una relación que se ve truncada cuando su padre decide mandarlo a Portugal a estudiar. Muchos años después, las vidas de Blas y su primer amor se cruzan en dos ocasiones, pero bajo circunstancias muy distintas a las de su idilio amoroso. La primera vez se presenta el mismo día en que Blas asiste a una reunión para conocer a Virgilia, la novia escogida por su padre para él; camino a la cita, Blas rompe el vidrio de su reloj 
y entra en un negocio en busca de una refacción, donde se encuentra con su antigua pareja sentimental. Pero Marcela ya no es la de antaño, pues su rostro se encuentra lleno de arrugas y de marcas de viruela. Los antiguos amantes sostienen sólo una breve conversación, pues Blas se siente incómodo y no quiere darle mayor importancia al asunto. Hacia el final de la novela, los caminos de Blas y Marcela se cruzan por tercera y última vez, cuando el primero, quien goza, aun sin trabajar, de una buena situación económica, reparte limosnas en el hospital de la Orden y ve a Marcela "fea, flaca y decrépita” (245) morir en ese mismo establecimiento.

La historia entre Marcela y nuestro cínico narrador encierra, sin embargo, una mayor complejidad de lo que este breve resumen permite entrever. Al describir la fiesta en casa de Marcela en la que Blas le roba un beso, advertí que ella no opuso resistencia. En realidad, la reacción de Marcela es fácil de explicar: ella es una meretriz que busca hombres adinerados y Blas es un buen prospecto de cliente. En el capítulo XIV, antes de relatar las oscuras circunstancias que le permitieron intimar con Marcela, el protagonista hace su autorretrato donde destaca no sus cualidades, sino su poder adquisitivo: "Sí, yo era un lindo muchacho, garboso y elegante, y fácilmente se imagina que más de una dama haya inclinado ante mí la frente pensativa, o levantado hacia mí sus ojos codiciosos” (44). A partir de esta autovaloración en términos comerciales el léxico de los siguientes capítulos ("El primer beso", "Marcela"), se torna mercantil:

Era una buena moza [Marcela], alegre, sin escrúpulos, algo cohibida ante la austeridad de la época, lo que no le impedía arrastrar por la calle, y en berlina, sus graciosas locuras; lujosa, impaciente, amiga del dinero y los muchachos. En aquel año, moría de amor por un cierto Javier, sujeto acomodado y tísico: una perla (45; las cursivas son mías).

Como se observa, la "austeridad de la época" contradice el lujo que rodea a la cortesana. Para mantener ese estilo de vida derrocha- 
dor, Marcela necesita que sus clientes sean pudientes, es decir, unas "perlas". En consecuencia, es lógico que Marcela haya recibido con gran interés (afectivo y económico) aquel primer beso. Para nuestro protagonista, tocar los labios de Marcela significó convertirse en su beneficiario económico: "Era mío el universo, pero - jay desdicha!- no era gratuito. Me fue preciso adquirir dinero, multiplicarlo, inventarlo" (47). Pero un hombre como él, hijo del ocio en muchos sentidos, es incapaz de producir riqueza:

Primero exploté la generosidad de mi padre; él me daba todo lo que le pedía, sin represión, sin demora, sin frialdad. [...] Pero, a tal extremo llegó el abuso, que él restringió un poco sus larguezas, luego más y más... Entonces recurrí a mi madre, y la induje a desviar alguna cosa, que me la daba a escondidas. Era poco; eché mano a un último recurso: pedí a cuenta de la herencia de mi padre, firmé obligaciones, que debía rescatar un día con usura (47; las cursivas son mías).

Existe aquí una acumulación de términos relacionados a una economía bastante improductiva, por no decir ocio y corrupción ("desviar", "recurrir", "abusar" y "pedir"). Lo que también queda claro es que a Blas jamás le cruza por la mente la idea de ponerse a trabajar como medio para obtener dinero.

La relación entre los amantes tiene su sustento en esos "antiguos doblones de oro" (47) que tanto le gustan a ella y suele agradecer con sus servicios, corroborando que el deseo sexual de Blas siempre va acompañado del factor económico y de la degradación de la amante en turno. Lo primero que se destaca de Marcela es su origen (espanola) y su belleza ("vistosa", "cuerpo esbelto", "linda Marcela" [45] y "hermosa" [50]). No obstante, estos calificativos sólo son válidos mientras Marcela conserve su riqueza y su estilo de vida dedicado al ocio, es decir, a la actividad improductiva. En cuanto dichas condiciones dejan de prevalecer, decae la belleza de la española, como acaece en los posteriores encuentros de estos personajes. En el episodio 
dentro del negocio, a donde Blas acude para reparar su reloj, resalta, ante todo, la fealdad de la que alguna vez ostentó el título de la más bella dama de la ciudad:

Al fondo, detrás del mostrador, estaba sentada una mujer, cuyo rostro amarillo y variloso no se destacaba a primera vista. [...] la enfermedad y una vejez precoz le destruyeron la flor de las gracias. Las viruelas habían sido terribles; las marcas, muchas y grandes, hacían salientes y muescas, subidas y pendientes, y daban una sensación de lija gruesa. [...] El pelo parecía tostado, y casi tan polvoriento como las puertas del negocio (88).

La descripción de una Marcela pobre y fea contrasta con las anteriores. Pero su degradación no ha llegado a su fin. Anteriormente la española solía recibir obsequios de sus múltiples admiradores y clientes. ${ }^{3}$ El protagonista también contribuye considerablemente a la colección de joyas de la meretriz: "-¡Pero, chico -me decía Marcela, cuando yo le llevaba alguna seda, alguna joya-, tú quieres reñir conmigo!... ¿ ¡Por qué haces esto?... ¡Un regalo tan caro!...” (47). Sin embargo, ahora detrás del mostrador, Marcela se ve obligada a pasar por una segunda afrenta, ${ }^{4}$ que se concreta cuando le pregunta a Blas si desea comprar algo. La dama que solía vivir de las compras ajenas, tiene la necesidad de vender (no su cuerpo, pues éste ya no tiene valor de cambio) para sobrevivir. Como en el caso de Eugenia, la crueldad del protagonista no conoce límites, pues Blas relata que el negocio de Marcela le fue heredado por uno de sus ex amantes: "Un hombre, que la amara otrora, murió en sus brazos, dejándole aquel negocio de platería; pero, para que la desgracia fuese completa, su clientela

${ }^{3}$ Casi todos los objetos que adornan su casa son dádivas recibidas a cambio de sus servicios sexuales. Blas, quien siente celos al verlos, los llama "despojos de sus amores de antaño" (48).

${ }^{4}$ La primera humillación consiste en la pérdida de su belleza. 
era muy escasa ahora, quizá por la singularidad de que el negocio lo dirigiera una mujer" (89). Las palabras de Blas entrañan un malicioso juego verbal. En primer lugar, se destaca que su ex cliente no le dejó joyas o dinero, sino un negocio, es decir, la puso a trabajar. ${ }^{5}$ Otra palabra clave es "clientela". La española se gana la vida vendiendo su platería a clientes esporádicos, pero ya sabemos que el vocablo "clientela" tiene, en el caso de Marcela, fuertes connotaciones sexuales. Como prostituta, Marcela dependía de su "clientela" masculina para sustentarse. Sin embargo, ahora es una mujer tan fea que en lugar de ver su casa adornada con obsequios, ella es la que provee a los hombres con joyas que servirán como regalos para las mujeres bellas del momento. Finalmente, la imagen de la decadencia de la ex prostituta queda rematada con la última palabra de la cita: "mujer". En opinión de Blas una de las razones de la falta de clientela tiene su origen en que una mujer dirija dicho establecimiento, insinuando que la única forma en que una mujer de su clase se convierta en una mujer de negocios es en la cama de los hombres ricos.

El proceso de humillación se concentra no sólo en la pérdida de belleza o de bienes materiales, sino también en el carácter. La primera vez que Blas alude a Marcela utiliza dos adjetivos singulares que jamás empleará con otras damas: “impaciente” y "arrogante" (45). Recordamos a la española en su espléndida casa dando órdenes a un paje que la obedece al pie de la letra. Asimismo, ella se regodeaba provocándole celos a su nuevo cliente: "Y, como se daba cuenta de mis celos tardíos, parecía que gozara en aguijonearlos más” (48). Pero quizá lo que ejemplifique con mayor claridad la antigua arrogancia de Marcela y su inicial posición de poder frente a Blas sea su actitud frente a los insistentes ruegos de su enamorado de acompańarlo a Portugal:

\footnotetext{
5 Etimológicamente "negocio" quiere expresar la "negación del ocio", es decir, la necesidad del trabajo.
} 
[...] fui yo el que se arrojó a los pies de ella, contrito y suplicante; se los besé, le recordé aquellos meses de nuestra felicidad solitaria; le repetía los nombres queridos de otros tiempos, sentado en el suelo, con mi cabeza en sus rodillas, apretándole las manos. Jadeante, enloquecido, le pedí llorando que no me abandonara... Marcela me miró un instante, callados los dos, y luego me separó blandamente, con gesto de aburrimiento.

-No me fastidies -dijo (52).

Frente a la indiferencia de la cortesana, Blas queda desconsolado y vaga por las calles de Río. Ahora bien, la escena anterior contrasta con las palabras del narrador en el negocio de Marcela, durante su primer reencuentro:

Me dio una silla, y, con el mostrador de por medio, me habló extensamente de ella, de la vida que llevara, de las lágrimas que yo le hiciera verter, de los recuerdos, de los desastres, en fin de las viruelas que le destrozaron el rostro, y el tiempo, al que ayudó la enfermedad, adelantando su decadencia (89).

Ahora ya no es Blas quien llora, sino Marcela; ya no es Blas quien rememora tiempos pasados, sino Marcela; ya no es Blas quien se lamenta de su suerte, sino Marcela. Los papeles han quedado invertidos. En esta nueva situación de poder el narrador dice, parodiando la respuesta que años antes recibió: "Marcela suspiró con tristeza. La verdad es que yo me sentía afligido y fastidiado, al mismo tiempo, y ansiaba verme fuera de esa casa" (90; las cursivas son mías). ${ }^{6}$ Marcela ha quedado desprotegida ${ }^{7}$ y enfrenta una situación catastrófica. Más allá de las marcas de las viruelas, la verdadera enfermedad de Marcela

\footnotetext{
${ }^{6}$ En el original portugués, la respuesta de Marcela es la siguiente: "Não me aborreça". Y, de igual manera, Blas se vale de la misma palabra para mostrar su despecho por ella: "A verdade é que eu me sentía pungido e aborrecido, ao mesmo tempo, e ansiava por me ver for a daquela casa".

7 “[Ella] deseaba tener la protección de los amigos de otro tiempo" (90).
} 
es no gozar de la protección y del favor de un rico. Las laceraciones físicas le permiten a Blas resolver una contradicción que se le presentó en el caso de Eugenia. En aquella ocasión el protagonista no entendía cómo era posible que existiera una persona bonita, pero pobre; con Marcela queda ese dilema resuelto, pues ella es ahora pobre, "fea, flaca" y "decrépita" (245).

Si Marcela representa el primer gran amor del protagonista, Eulalia Damasceno, alias Loló, es su última oportunidad para acceder al matrimonio. De igual forma que con Virgilia, Blas, ahora cuarentón, conoce a Loló a través de un intermediario que se ha propuesto casarlo, su hermana Sabina. En un primer encuentro, Blas se muestra indiferente hacia la joven, pero para el segundo, los encantos de Loló comienzan a tener un efecto positivo sobre el solterón, al grado de que decide comenzar un noviazgo con ella. La relación marcha tan bien que Blas, persuadido por Sabina, toma la decisión de contraer nupcias, sin embargo, Loló fallece repentinamente, víctima de una epidemia. De esta manera Blas evita una vez más el matrimonio.

$\mathrm{Al}$ comentar la estructura social brasileña en las últimas décadas del XIX, Schwarz asevera que "It is no exaggeration therefore to state that the country's social structure itself privileged the mechanism of the personal favor, including its inevitable -and by the late nineteenth century, unacceptable-component of whim" (1996: 168). Una de las manifestaciones de dicho capricho de voluntad y de poder social podía presentarse como "the 'lucky break' of cooptation (in this case, an unequal marriage), possibly coupled with the humiliation of dependence" (1996: 168). Contraer nupcias con Loló representaría para Blas unir su vida con alguien de una clase social inferior. "Me dispuse a aceptar todo, hasta la alianza de Damasceno" (204), comenta Blas después de ceder a la propuesta de su hermana. La palabra cargada de mayor sentido es "hasta", pues en ella se encierra todo un mundo de diferencias sociales. El narrador, en realidad, presenta el caso como si él le hiciera un "favor" a Loló al casarse con ella. 
De Marcela sabemos sólo que su padre fue un hortelano, de Eugenia se insinúa maliciosamente la posibilidad de que su progenitor fuese una aventura fugaz de dońa Eusebia. En cambio, Damasceno es el único padre de las amantes de Blas que llegamos a conocer relativamente bien y podemos concluir que se trata de un hombre que se propone medrar socialmente vendiendo a su hija al mejor postor. Desde un inicio, Damasceno quiere familiarizar a Blas con los más mínimos detalles de su vida familiar, donde se pueden destacar cuatro aspectos: a) la cultura que desea aparentar; b) las supuestas virtudes de su hija; c) su odio a los ingleses; y d) un afecto sin sustento por Blas. Damasceno le refiere al narrador sus visitas al teatro citando el título de dramas, comedias y óperas. Asimismo aprovecha la menor oportunidad para presentar a su hija bajo una extraordinaria luz: "Ahora quería oír Ernani, que su hija cantaba en casa al piano: -Ernani, Ernani, involami... [...] La hija se moría por oír todas las óperas. Tenía una voz delicada la hija. Y gusto, mucho gusto" (170). Antes de siquiera hacer su aparición como personaje, Loló es presentada por su padre como una mujer que disfruta los gustos de la alta sociedad. Otro tema que apasiona a Damasceno es su odio por los ingleses. ${ }^{8}$ Finalmente, Damasceno termina por ponerse en ridículo al mostrar un afecto sin fundamentos empíricos por el protagonista. ${ }^{9}$ La simpatía de Damasceno por Blas tiene su única razón en su intención de casar a Loló con un adinerado cuarentón, lo cual le permitiría escalar posiciones sociales por medio de un matrimonio arreglado.

Sabemos que Blas justifica a través de un proceso sistemático de humillación su decisión de terminar su corto romance con Eugenia;

\footnotetext{
8 “QQue se fueran al diablo los ingleses! Esto no se enderezaría mientras no se los echara a todos. ¿Qué podría hacernos Inglaterra?” (170).

9 "Lo llevé hasta la puerta de la sala; él se detuvo, diciéndome que simpatizaba mucho conmigo" (171).
} 
la relación con Marcela recorre el mismo camino. Sin embargo, el caso de Loló no se rige del todo bajo esa dinámica. Por un lado, Blas hace, poco a poco, de Loló una mujer socialmente aceptable, pero, por otro, resalta algunos de sus defectos para poder justificar un posible abandono, si éste fuese necesario, o para establecer una relación de dependencia hacia él. Esta actitud ambivalente se manifiesta en dos términos: "elegante" y "ojos". Con la primera palabra se alude al proceso de refinamiento de Loló y, con la segunda, a la forma de ser ofrecida por su padre como una mercancía a la venta. Veamos esto con más detalle.

Blas hace coincidir en una oración los dos vocablos de marras, al describir sus primeras impresiones de Loló: "Felizmente, Sabina me hizo sentar al lado de la hija de Damasceno, una tal Eulalia, o más familiarmente Loló, muchacha graciosa, un tanto cohibida al principio, pero sólo al principio. Le faltaba elegancia, mas la compensaba con los ojos" (171). Aunque Loló se esmera por agradar a Blas, cantando bellamente, Blas no la encuentra a su altura; pero cuando varias noches después Blas la ve en el teatro cambia su opinión: "Vestía la hija con otra elegancia y cierto esmero, cosa difícil de explicar, porque el padre ganaba apenas lo necesario para endeudarse; quizás fuera por esto mismo" (176). La "otra elegancia" de Loló no consiste en su nuevo vestido, sino en la disponibilidad de Damasceno para endeudarse. Por una parte, el vestido le otorga un valor a Loló que no le brinda su refinada voz y, por otra, enfatiza la futura situación de dependencia de la joven en caso de un matrimonio. Ambas cosas agradan al protagonista y crean completamente una nueva situación:

Parecía más bonita que el día de la comida. Le hallé cierta suavidad etérea, unida al refinamiento de las formas terrenas; expresión vaga y condigna de un capítulo en que todo ha de ser vago. Realmente, no sé cómo decirte, lector, que no me sentí mal junto a la joven, luciendo elegantemente un lindo vestido, un vestido que me daba cosquillas de Tartufo (176s.). 
Está claro que elegancia significa la aceptación de la superioridad social de Blas por parte de Loló. De esta manera, a ojos de Blas, la hija de Damasceno no comete los mismos "errores" que Eugenia, y es aceptable para un noviazgo. ${ }^{10} \mathrm{~A}$ Loló no le interesa borrar las barreras sociales que existen entre Blas y ella, sino, más bien, mostrar su capacidad de hacerse pasar por alguien de una clase social más alta, aunque esto implique su aceptación de que jamás dejarán de existir dichas barreras, exactamente lo que no quiso hacer Eugenia. En consecuencia, Loló se empeña en aprender la "elegancia" e imitarla: "La vida elegante y refinada la atraía, principalmente porque le parecía el medio más seguro de amoldar nuestras personas. Loló observaba, imitaba, adivinaba, esforzándose al mismo tiempo en disfrazar la inferioridad de la familia" (207). Esta actitud le posibilita a la mujer anular la mala impresión que dio a Blas el día en que se conocieron y transformarla en una positiva, casi eufórica, pues el narrador la eleva al grado de ser "tierna, luminosa, angelical" (205), lo que confirma que en el mundo cínico de Blas Cubas un matrimonio desigual es admisible en tanto confirme el poder de la clase dominante.

\footnotetext{
${ }^{10}$ Recordemos que uno de los primeros "errores" de Eugenia se centra en su vestimenta. Invitado a comer a casa de dońa Eusebia, Blas ve a Eugenia por segunda ocasión, pero la muchacha viste de forma sencilla y no porta joya alguna: "Un simple vestido blanco, de casa, sin adornos, teniendo en el cuello, en vez de broche, un botón de nácar, y otro botón en los puños, cerrando las mangas, y ni sombra de pulsera" (81). Se han sugerido dos interpretaciones posibles para esta actitud inusual de Eugenia. Por un lado, Eugenia estaría aceptando la diferencia de clase entre ella y Blas e indicando con ese vestido que renuncia a cualquier fantasía de paridad social. O por otro lado, en una lectura menos ingenua, Eugenia estaría recordándole a Blas que los hombres, juzgados desde su interioridad y no por sus prendas de vestir, son todos iguales: "However, it seems clear that [Eugênia's] actions can be read differently: by doing away with external ornamentation, she reminds her visitor that individuals are fundamentally equal and implicitly forbids him to treat her as an inferior" (Schwarz, 1996: 166). Las consecuencias de esta posición son conocidas al lector; Blas humilla y destruye moralmente a Eugenia.
} 
Según se observa, la "elegancia" cumple un papel primordial en el proceso de revaloración de Loló y en la autoaceptación de su dependencia. Ahora bien, la joven también es objeto de un proceso de degradación al cual no sólo contribuye Blas, sino, sobre todo, su propio padre. Dicho proceso está íntimamente relacionado con sus ojos desde el primer encuentro: "Le faltaba elegancia [a Loló], mas la compensaba con los ojos, que eran soberbios, y sólo tenían el defecto de no arrancarse de mí, excepto cuando bajaban al plato; pero Loló comía tan poco que casi no miraba al plato" (171). Si bien la actitud de Loló es grotesca, también es efectiva, pues llama la atención de Blas. La joven no está interesada en la comida, pero sí en el rico soltero, a quien se come con los ojos. Durante su segundo encuentro en el teatro, Loló insiste en su estrategia: "Entro; recorro los palcos con la vista; en uno de ellos veo a Damasceno y familia. [...] En el intervalo fui a visitarlos. Damasceno me recibió con muchas palabras, su mujer con muchas sonrisas. En cuanto a Loló no me sacó los ojos de encima" (176). Así como Loló no comete el error de Eugenia de elegir una vestimenta de poca etiqueta, tampoco lo hace en relación a no cumplir las expectativas de Blas, quien necesita ser admirado y observado por las mujeres. ${ }^{11}$ Loló, al no separar sus ojos de la figura del protagonista, alimenta el orgullo de Blas y se gana parte de sus simpatías.

Sin embargo, como ya se ha señalado, el motivo de los ojos también guarda una connotación negativa. Dentro de la dinámica de la

\footnotetext{
${ }^{11}$ Precisa recordar la actitud de Eugenia; el mismo día en que ella y Blas se conocen ocurre un fugaz segundo encuentro: "Por la tarde, vi pasar a caballo a la hija de dońa Eusebia, seguida de un paje; me hizo un saludo con la punta del chicote. Confieso que me lisonjeó la idea de que, algunos pasos adelante, ella volvería la cabeza hacia atrás; pero no la volvió" (78). Blas es herido por Eugenia en su orgullo egocéntrico y masculino, lo que acelera su decisión de acabar "de una vez con esta flor de la mata" (84).
} 
novela, dicho motivo en combinación con la figura de Damasceno sirve para reafirmar su calidad de objeto lucrativo. En el capítulo 121, Blas relata su descenso, en compañía de Damasceno y Loló, de la capilla de la Liberación que se encuentra sobre el morro, donde se topan con un grupo de "hombres de todas las edades, tamaños y colores, unos en mangas de camisa, otros de chaqueta, otros enfundados en levitas raídas; [...] todos con los ojos fijos en el centro y brillándoles el alma en las pupilas" (205). Lo que esta variopinta masa, formada por mulatos y esclavos, observa es una pelea de gallos. Loló y Blas se alejan, a iniciativa del segundo, rápidamente del sitio. Damasceno, en cambio, fascinado por el espectáculo, interactúa con aquellos hombres de baja calaña, provocándole una enorme vergüenza a su hija, pues "la facilidad con que [Damasceno] familiarizara con los apostadores ponía de relieve antiguos hábitos y afinidades sociales" (207). Sin embargo, lo que interesa es cómo la pelea de gallos permite una asociación, por medio del motivo de los ojos, entre Loló y los gallos y, por extensión, entre Damasceno y los apostadores:

El centro les tenía atados los ojos. Era una rińa de gallos. Vi a los dos contendores, dos gallos de espolón agudo, ojo de fuego y pico afilado. Ambos agitaban las crestas ensangrentadas; el pecho de uno y de otro, desplumado y rojo; les invadía el cansancio. Pero luchaban aun así, los ojos fijos en los ojos, pico abajo, pico arriba, golpe de éste, golpe de aquél, vibrantes y rabiosos. Damasceno no sabía nada más; [...] La riña de gallos era una de sus pasiones (206; las cursivas son mías).

La atención que se pone sobre los ojos de los espectadores y de los animales es insistente. Ahora bien, el único personaje que recibió a lo largo de la novela una mención sistemática de sus ojos es Loló, lo cual invita a suponer, por medio de una asociación verbal, un destino común entre mujer y animal, al ser ambos utilizados para obtener ganancias económicas. Loló se convierte en el gallo de Damasceno, al que le apuesta su futuro. Por ańadidura, en esta escena queda pre- 
figurada la muerte de Loló. Observamos, entonces, cómo Machado de Assis dotó al episodio de Loló de unidad interna a partir de la frase "Le faltaba elegancia, mas la compensaba con los ojos", pues ésta se disemina a lo largo de varios capítulos, tejiendo una telarańa de asociaciones.

La historia de amor entre Virgilia y Blas es, a su vez, la que ocupa el mayor número de páginas del libro. Hay una desproporción evidente entre la extensión de esta relación con las otras tres mujeres. Mientras que unos cuantos meses son el marco de los noviazgos de Blas con Marcela y Loló, o sólo un par de semanas en el caso de Eugenia, su relación amorosa con Virgilia abarca más de una década. La razón de tan prologando romance hay que buscarla en la pertenencia de Virgilia a una clase social alta, cuya familia tiene incluso más poder político que la de Blas.

Precisa destacar ciertas coincidencias y divergencias de las relaciones previamente analizadas con la de Virgilia. Un factor común a todas las relaciones amorosas del protagonista son los múltiples reencuentros después de varios años. Normalmente, Blas narra sus memorias siguiendo la estricta cronología de su vida. No obstante, el caso de Virgilia es una excepción debido a que comienza con el libro y, como se sabe, el inicio de la novela coincide con el fin de la vida de Blas. Así, el primer diálogo entre Virgilia y Blas se refiere, en realidad, a su último reencuentro cuando Blas yace en su lecho de muerte. En consecuencia, el lector conoce desde un principio el desenlace de esta larga historia de amor clandestino, a diferencia de lo que acaece con los otros romances.

$\mathrm{Al}$ igual que con las otras mujeres, Blas llama al inicio a Virgilia "fresca", "juvenil" y "florida" (94) y al final la presenta como una "imponente ruina" (18). Virgilia es tan codiciosa y ambiciosa como Marcela y casi tan joven como Eugenia, con quien comparte que Blas piense en ella como futura lectora: "Tú que me lees -si vives aún cuando estas páginas vean la luz-; tú que me lees, Virgilia amada, 
¿no reparas en la diferencia entre el lenguaje de hoy y el que primero empleé cuando te vi?” (73). ${ }^{12}$ Virgilia, como Loló, conoció a Blas a través de un intermediario, el padre del protagonista que la eligió como futura esposa de su hijo.

Pero más allá de las coincidencias persisten ciertas divergencias irreconciliables. La relación entre Virgilia y Blas jamás alcanza los niveles pasionales de las otras tres; en realidad se trata de una relación de mediocres tintes románticos. ${ }^{13}$ Por otra parte, aunque de Virgilia conocemos sus debilidades, sus defectos, su adulterio e incluso su vejez, Blas no se aprovecha de estas circunstancias para humillarla. $\mathrm{O}$ dicho de otra manera: a Virgilia le sobra la elegancia, que tanta falta le hacía a la joven Loló, pero le falta el grado de sensualidad propio de las mujeres de clase inferior que tanto atraían sexualmente al protagonista.

Cuatro son las mujeres con las que Blas Cubas se relaciona a lo largo de su vida. Tres de ellas pertenecen a una clase social inferior a la suya con la excepción de Virgilia. Asimismo es evidente que existe una correspondencia íntima entre los episodios de Loló, Eugenia y Marcela, que contribuye a dotar a las Memorias de una unidad interna, de la cual pareciera carecer a causa de su estructura episódica. La unidad de esta novela se refleja en sus imágenes, en sus motivos y, en gran parte, en el tono del narrador.

Si esta tríada de mujeres sirve para mostrar cómo Blas se relaciona con personas de las clases inferiores, ¿no hubiera bastado entonces un solo ejemplo? Hay que responder que pese a sus conexiones claras, no se trata aquí de presentar una misma situación. Como en toda variación, lo destacable no es el motivo que permanece, sino la novedad

12 A Eugenia le había dicho: “Tú, mi Eugenia, no te descalzaste nunca” (86).

${ }^{13}$ Sobre una interesante valoración del triángulo amoroso formado por Blas, Virgilia y el marido cornudo, Lobo Neves, cfr. Schwarz, 1990: 127. 
que se introduce. Por otro lado, dichas variaciones permiten mostrar el desarrollo interno del personaje. Las relaciones con Marcela, Loló y Eugenia se agotan casi en el mismo momento en que se inician, porque ningún hombre de la clase dominante se iba a relacionar por largo tiempo con una mujer de clase inferior, a menos de que fuera a través de un improbable matrimonio. Además, hablar de las mujeres de Blas Cubas es hablar, en realidad, de él, quien es un hombre con una gran tragedia sobre sus espaldas, al ser incapaz de amar. Una de las proezas de las Memorias póstumas de Blas Cubas, y no la más pequeña, es presentar un tema tan cruel y tan trágico como una comedia llena de humor, donde el lector parece, a veces, olvidarse de que en este libro la risa sustituye al llanto, proponiendo una estética tragicómica que en el resto de América Latina sólo empezaría a tener sus primeros representantes cuarenta años después con Roberto Arlt en Argentina y que la literatura mexicana tardaría varias décadas más en empezar a hacer suya.

\section{Bibliografía}

Machado de Assis, J.M., 1940, Memorias póstumas de Blas Cubas, versión castellana de Francisco José Bolla, Club del Libro/A.L.A., Buenos Aires.

Schwarz, Roberto, 1990, Um mestre na periferia do capitalismo: Machado de Assis, Duas Cidades, São Paulo.

, 1992, Ao Vencedor as Batatas. Forma literária e proceso social nos inícios do romance brasileiro, Duas Cidades, São Paulo.

, 1996, "The Historical Meaning of Cruelty in Machado de Assis" en Modern Language Quarterly 57: 2, pp. 165-179. 